\title{
A MODEL STUDY OF POWDER-SNOW AVALANGHES
}

\author{
By E. J. Hopfinger and J.-C. Tochon-DanguY \\ (Institut de Mécanique, Laboratoire associé au C.N.R.S., Université de Grenoble, B.P. 53, \\ Centre du Tri, 3804I Grenoble-Cédex, France)
}

\begin{abstract}
A powder-snow avalanche is a particular type of turbulent gravity current which it is proposed to treat using methods developed in contexts where the Boussinesq approximation is valid. Hence, it is assumed that the air entrainment rate $E$ primarily depends on the internal Froude number (Tochon-Danguy and Hopfinger, [1975]) and that the large density variation in an avalanche reduces only the drag resulting from the air entrainment. In order for this drag to dominate over the wall stress it is necessary that the slope angle be greater than a certain minimum value which depends on the density ratio of the flow. For a lowdensity flow, for example, a minimum slope angle of about $5^{\circ}$ is necessary. The more unusual aspect is that an avalanche may also entrain snow along its path which increases its mass and can produce in certain cases an accelerating flow. This aspect is included in the theoretical treatment. Experiments have been carried out in an inclined water channel using brine as dense fluid. Visual observations as well as measurements of the velocity and density profiles have been made. Various states of flow are proposed depending on whether the avalanche is "long" (having the structure of a usual gravity current) or "short" (having the structure of a thermal) or whether snow is entrained. The practical consequences which can be drawn from these results are discussed, in particular in the context of the design of defence structures, and flow visualizations of a gravity current impinging on an obstacle give an idea of the protected region.
\end{abstract}

RÉsumé. Une étude modèle des avalanches de neige poudreuse. Une avalanche de neige poudreuse est un type de courant de gravité turbulent et il est proposé de l'aborder en utilisant les méthodes développées dans le contexte des écoulements permettant l'approximation de Boussinesq. De ce fait, il est supposé que le taux d'entraînement d'air $E$ dépend principalement du nombre de Froude densimétrique (Tochon-Danguy et Hopfinger, [1975]) et que la forte variation de densité dans une avalanche a comme effet de réduire la trainée dûe à cet entrainement. Pour que la trainée d'entrainement d'air domine sur le frottement au sol, il est nécessaire que l'angle de la pente du terrain soit supérieure à une certaine valeur critique qui est fonction du rapport des densités. Pour une situation de faible densité, par exemple, une pente de $5^{\circ}$ est nécessaire. L'aspect le plus inhabituel est qu'une avalanche peut également entraîner de la neige qui augmente sa masse et peut avoir pour effet un écoulement qui s'accélère d'une manière continue. Le traitement théorique présenté ici tient compte de cet entraînement. Les expériences ont été effectuées dans un canal incliné utilisant de l'eau salée au-dessous de l'eau douce. Des observations visuelles ainsi que des mesures des profils de vitesse et de densité ont été faites. Plusieurs états de mouvement sous proposés. Ainsi l'avalanche peut être "longue" (ayant la structure d'un courant de gravité habituel) ou "courte" (ayant la structure d'une bouffée flottante) ou régie par l'entraînement de neige. Les conséquences pratiques qu'on peut tirer de ces résultats sont discutées, notamment dans le contexte des ouvrages de protection passive et des observations visuelles d'un courant de gravité frappant un obstacle donnent une idée de la zone protégée.

Zusammenfassung. Eine Modellstudie für Pulverschnee-Lawinen. Eine Pulverschnee-Lawine ist ein besonderer Typ eines turbulenten Schwerkraftstromes. Es wird deshalb vorgeschlagen, sie nach Methoden zu behandeln, die für Vorgänge entwickelt wurden, wo die Boussinesq-Näherung gültig ist. Folglich wird angenommen, dass die Luftaufnahmerate $E$ vor allem von der inneren Froude-Zahl abhängt (Tochon-Danguy und Hopfinger, [1975]) und dass die grosse Dichteschwankung in einer Lawine nur die Bremsung infolge des Luftgehaltes reduziert. Für ein Überwiegen dieser Bremsung über den Druck an den Uferwänden ist es notwendig, dass die Hangneigung einen gewissen Mindestbetrag überschreitet, der vom Dichteverhältnis des Stromes abhängt. Bei geringer Stromdichte ist zum Beispiel eine Mindesthangneigung von etwa $5^{\circ}$ erforderlich. Ungewohnter ist der Gesichtspunkt, dass eine Lawine entlang ihrer Bahn auch Schnee aufnimmt, wodurch ihre Masse vergrössert und in bestimmten Fällen ein beschleunigter Fluss erzeugt wird. Dieser Gesichtspunkt ist im theoretischen Ansatz berücksichtigt. Versuche wurden in einem geneigten Wassergerinne mit Salzlauge als dichter Flüssigkeit durchgeführt. Es wurden visuelle Beobachtungen wie auch Messungen der Geschwindigkeit und der Dichteprofile vorgenommen. In Abhängigkeit davon, ob die Lawine "lang" (mit einer Struktur wie ein gewöhnlicher Schwerkraftstrom) oder "kurz" (mit einer Struktur wie ein Wärmestrom) ist oder ob Schnee aufgenommen wird, lassen sich verschiedene Fliesszustände annehmen. Die praktischen Folgen dieser Ergebnisse werden erörtert, insbesondere im Hinblick auf Lawinenverbauungen, und Fliessvorstellungen über einen Schwerkraftstrom, der an ein Hindernis anprallt, geben Anhaltspunkte über das geschütze Gebiet.

\section{InTRODUCTION}

Field observations of avalanches are cumbersome, extremely costly, and necessarily limited. Although there is little doubt about the importance of such observations it is desirable to complement or replace them when feasible with laboratory studies. It seems indeed to be a very difficult and ambitious task for instance to try to measure the velocity and density 
distributions in a powder-snow avalanche, information which is, however, crucial for an efficient barrier design. At present the dimensions of these defence structures are determined on the basis of impact-pressure measurements which are themselves incomplete and are quite often difficult to interpret. The first results of laboratory studies (Tochon-Danguy and Hopfinger, [1975]), referred to from hereon as (I), led us to believe that an adequate simulation (that is, giving quantitative results), of certain types of avalanches is possible, and that even the use of scale models may be considered. Such an attempt was made by Lovie and others (1974) who made a I/5 ooo model of the Northern Peclet Glacier, in Val Thorens. They were principally interested in determining the avalanche path for different points of release in order to determine whether or not it would be safe to build a restaurant at a certain site. The authors drew decisive conclusions on the basis of their model study. It must be said, however, that the reliability of their results is doubtful, mainly because the Reynolds number of the flow is too small and hence the simulated currents can hardly represent powdersnow avalanches. The path of powder-snow avalanches may be quite different from that of a flood-wave type flow. Furthermore, the scaling applied to the velocities was incorrect.

Powder-snow avalanches have a Newtonian behaviour and this facilitates their simulation. It has been shown in (I), that when the Reynolds number is sufficiently high, similarity is assured when, besides the boundary conditions, the densimetric Froude number as well as the ratio of the current to the ambient fluid density are the same. If both parameters were respected, the only free variable at our disposal is the height of the current and this means that the velocity in the laboratory (imposed by the Froude number) is too high to be practical, that is to say in order to reach an established flow the channel dimensions would have to be considerable. A distortion in the density ratio is therefore desirable. In the laboratory a salt solution or a suspension of sand in water with a density ratio ranging from I.OI to I.I 5 is used, whereas in a powder avalanche this ratio is more of order Io. A flow satisfying the Boussinesq approximations is thus used to simulate a flow where such an approximation is not justified. This is of some consequence when considering the effect of the entrainment of ambient fluid, but the structure of the flow is believed not to be appreciably affected.*

The key argument was that for the structure of the laboratory gravity current to be similar to an avalanche, the momentum transferred to the entrained air (which appears as interfacial stress) must dominate over the wall stress. The latter is a weak function of the Reynolds number whereas the air-entrainment rate is a function of the densimetric Froude number and thus increases with the slope angle. In the laboratory with $\rho_{\mathrm{a}} / \rho \simeq \mathrm{I}$ the wall friction becomes negligible at angles $\alpha>5^{\circ}$, whereas in an avalanche the entrainment effect is weighted by $\rho_{\mathrm{a}} / \bar{\rho}$ (with $\rho_{\mathrm{a}} / \bar{\rho} \approx 0.2$ to 0.5 ) and hence will become dominant only at a greater angle; its value is evaluated as about $20^{\circ}$ when taking $\rho_{\mathrm{a}} / \bar{\rho}=0.2$. In this evaluation a wall-friction coefficient of about $\mathrm{I} \times \mathrm{IO}^{-3}$ was used as compared with a value of about $3 \times 1^{-3}$ appropriate in the laboratory (Ellison and Turner, 1959). At greater slope angles the flow structure seems to become independent of the slope; only the rate of growth depends on it. In an avalanche this conclusion is probably only approximately valid, since the reference density is a function of $x$ and the entrainment rate is somewhat density dependent.

A salt-solution gravity current differs little from a suspension gravity current if besides incompressibility of the carrier fluid the terminal velocity of the solid particles is small compared with the velocity of the turbulent motion. In a powder-snow avalanche these conditions seem, in general, to be verified.

In (I) the global development of a gravity current on various slopes was considered. In this paper velocity and density profiles of such a current are given and the flow structure in the head has been determined from photographic records of the current taken with a moving

\footnotetext{
* Experiments with metal-powder suspensions are in preparation to verify this important conjecture; density ratios of about 2 may be reached with such suspensions.
} 
camera. Furthermore, the motion of a finite volume of dense fluid down a slope has been studied and the snow entrainment mechanism simulated. Some results obtained from visual studies of a gravity current when it hits or flows over obstacles which are models of avalanche defence structures are also discussed.

\section{Theoretical considerations}

\section{I. Steady gravity current}

For a two-dimensional gravity current flowing in the $x$ direction and with zero ambient pressure gradient, the momentum and continuity equations are:

$$
\rho\left(\frac{\partial u}{\partial t}+u \frac{\partial u}{\partial x}+w \frac{\partial u}{\partial z}\right)-g \frac{\mathrm{d}}{\mathrm{d} x} \int \Delta \rho \cos \alpha \mathrm{d} z-g \Delta \rho \sin \alpha+\rho \frac{\partial}{\partial z} \overline{u^{\prime} w^{\prime}}=0,
$$

and

$$
\left.\begin{array}{rl}
\frac{\partial u}{\partial x}+\frac{\partial w}{\partial z} & =0, \\
\frac{\partial \Delta \rho}{\partial t}+\frac{\partial \Delta \rho u}{\partial x}+\frac{\partial \Delta \rho w}{\partial z} & =0 .
\end{array}\right\}
$$

For the energy equation we have simply:

$$
\frac{\mathrm{D} S}{\mathrm{D} t}=\mathrm{o}
$$

where $S$ is the entropy. Here $z$ is normal to $x, g$ the gravitational acceleration, $\rho$ the local density of the mixture and $\Delta \rho=\rho-\rho_{\mathrm{a}}$, with $\rho_{\mathrm{a}}$ the ambient fluid density. When the Boussinesq approximation is valid, Equation (I) is a good approximation of the flow, whereas, in the case of an avalanche, terms containing the density fluctuations (snow concentration fluctuations) may not necessarily be small. Equation (2) is based on the incompressibility of the carrier fluid of density $\rho_{\mathrm{a}}$ which in general has the form (Soo, 1967, p. 259)

$$
\frac{\partial u_{j}}{\partial x_{j}}=\frac{\mathrm{I}}{\mathrm{I}-\phi}\left[u_{j} \frac{\partial \phi}{\partial x_{j}}-\frac{\partial \phi u_{\mathrm{p} j}}{\partial x_{j}}\right] \text {, }
$$

where $\phi$ is the volume fraction of the suspended solid material and $u_{\mathrm{p} j}$ the particle velocity. When $u_{j} \approx u_{\mathrm{p} j}$ Equation (4) reduces to Equation (2). For this to be true, the diameter of the suspended particles must be sufficiently small for the terminal velocity to be small compared to the characteristic turbulent velocity. The analysis presented in (I) is based on the validity of Equations (I) to (3) and the existence of a quasi-steady flow in which case $\partial / \partial t=0$.

Another important assumption is the incompressibility of the avalanche flow. It is clear that ahead of the avalanche front the application of the compression shock formula as used by some workers cannot be justified mainly because the air is not confined. On the contrary, in the snow cover ahead of the front the air trapped in it may be considered confined and thus could permit the build-up of a shock wave which would then disrupt the snow cover. Inside the avalanche, the sound velocity is likely to be lower than in the air due to the inertia of the suspended solid particles and hence the appearance of a shock is in principle more probable. A crude estimation of the sound velocity based on formulas given in Soo (1967, p. 237) led us to the conclusion that only in very unfavourable conditions would the sound velocity approach the fluid velocity and a weak quasi-stationary shock thus occur. The effects of the free surface of the flow and the non-uniform concentration are, however, likely not to favour shock formation. This aspect and the great uncertainty in the evaluation of the sound velocity are more in favour of the validity of the incompressibility assumption than the contrary. 


\section{2. "Inclined thermal"}

The flow of a finite volume of buoyant fluid down a slope, referred to as "inclined thermal", is a situation which may be as pertinent to avalanches as is the steady gravity-current situation, in particular when allowance is made for the snow entrainment from the powder-snow cover ahead of the avalanche. The front velocity of the two-dimensional inclined thermal shown schematically in Figure I can be written as:

$$
U_{\mathrm{f}}=C_{\mathrm{I}}\left(\frac{\Delta \rho}{\bar{\rho}} g A^{\frac{1}{2}} \sin \alpha\right)^{\frac{1}{2}},
$$

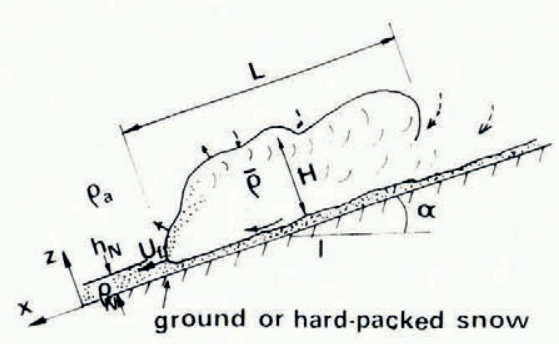

Fig. I. Sketch showing the characteristics of an "inclined thermal" with snow entrainment.

where $C_{\mathrm{I}}$ is a constant and $A \propto H L$ is the volume per unit width. For the definition of the other symbols see Figure $\mathrm{I}$. The variation of the excess mass of the thermal is given by:

$$
\frac{\mathrm{d}}{\mathrm{d} t}(\Delta \rho A)=C_{2} \Delta \rho_{\mathrm{N}} h_{\mathrm{N}} U_{\mathrm{f}},
$$

where ${ }_{\mathrm{c}}^{-} \Delta \rho_{\mathrm{N}}=\rho_{\mathrm{N}}-\rho_{\mathrm{a}}$. With $\mathrm{d} x / \mathrm{d} t=U_{\mathrm{f}}$ Equation (6) takes the form

$$
\frac{\mathrm{d}}{\mathrm{d} x}(\Delta \rho A)=C_{2} \Delta \rho_{\mathrm{N}} h_{\mathrm{N}}
$$

Integration of Equation (7) gives:

$$
\Delta \rho A=C_{2} \Delta \rho_{\mathrm{N}} h_{\mathrm{N}} x+\Delta \rho_{0} A_{0}
$$

where the subscript o refers to an initial state. Substitution of Equation (8) into Equation (5) gives:

$$
U_{\mathrm{f}}=C_{\mathrm{I}}\left[\frac{g \sin \alpha}{\bar{\rho} A^{\frac{1}{2}}}\left(C_{2} \Delta \rho_{\mathrm{N}} h_{\mathrm{N}} x+\Delta \rho_{0} A_{0}\right)\right]^{\frac{1}{2}} .
$$

Using similarity arguments we may write $A=S H^{2}$, where $S$ is a shape factor. Furthermore we may assume that $H$ is a linear function of the distance $x$. This assumption is valid (see section 4) when $h_{\mathrm{N}} \ll H$ and $\rho_{\mathrm{a}} / \bar{\rho} \approx \mathrm{I}$. The coefficient $E$ in $H=E x$ must be determined experimentally and in general $E=E\left(\alpha, \rho_{\mathrm{a}} / \bar{\rho}\right)$ but the dependence on $\rho_{\mathrm{a}} / \bar{\rho}$ is believed to be weak (see (I)). With these assumptions Equation (9) yields:

$$
U_{\mathrm{f}}=C\left[g \frac{\sin \alpha}{\bar{\rho} E}\left(C_{2} \Delta \rho_{\mathrm{N}} h_{\mathrm{N}}+\Delta \rho_{\mathrm{o}} A_{\mathrm{o}} / x\right)\right]^{\frac{1}{2}},
$$

which shows that the velocity of a finite volume of buoyant fluid decreases with distance as $x^{-\frac{1}{2}}$ when $h_{\mathrm{N}}=0$ and is constant and proportional to $h_{\mathrm{N}^{\frac{1}{2}}}$ when $h_{\mathrm{N}} \neq 0$. When $\Delta \rho / \bar{\rho} \simeq \mathrm{I}$ it is seen from Equation (5) that the velocity is proportional to $A^{\frac{1}{2}}$. An avalanche cloud is thus governed by Equation ( 10 ) only when the snow concentration is sufficiently low. The coefficients $E$ and $C$ can be obtained from laboratory experiments. 

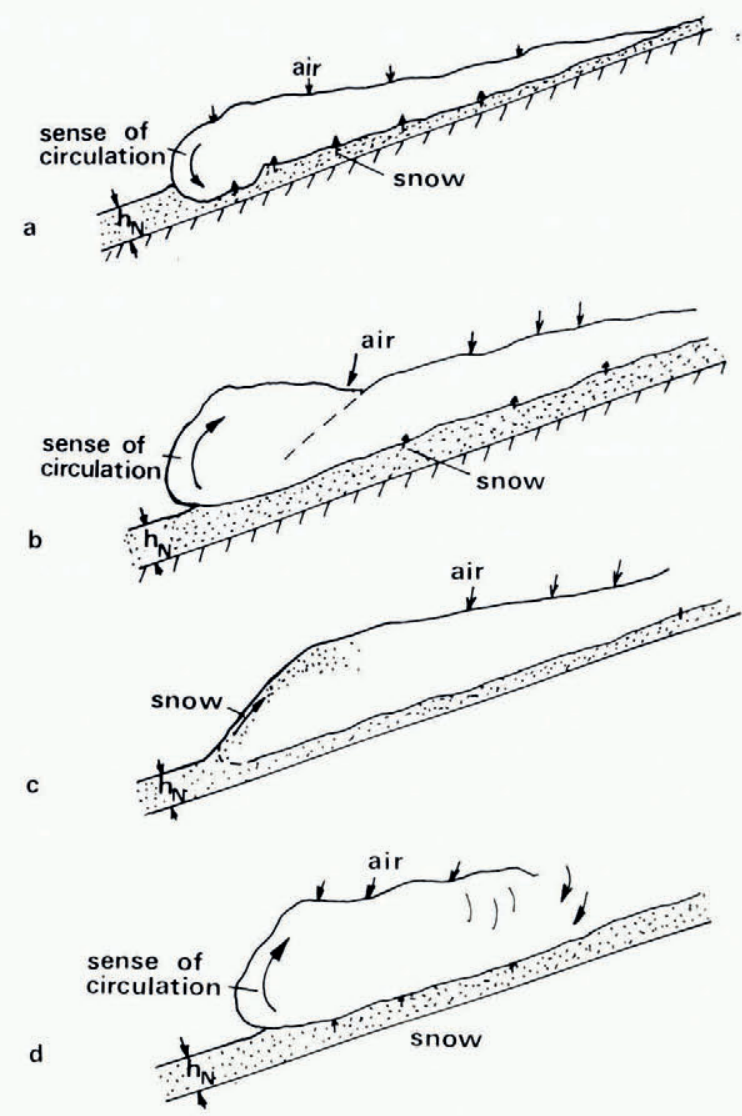

Fig. 2. Sketch showing the different possibilities of powder-avalanche motion (after the initial sliding): (a) gravity current with dominant snow-entrainment drag; $(b)$ gravity current with dominant air-entrainment drag; $(c)$ gravity current or thermal with dominant air-entrainment drag and entraining snow at its front; $(d)$ "inclined thermal" with dominant air drag.

At the start of avalanche motion, when $\bar{\rho} \approx \rho_{\mathrm{N}}$ and the momentum loss due to air entrainment is negligible, the structure of the motion is likely to be close to the starting plume model of Longuet-Higgins and Turner (1974) in which case the head erodes the snow cover. But as the current is diluted by the air entrainment process the structure changes gradually to that simulated in the laboratory, and Equation (10) will more closely approximate the flow. The different states of motion are shown schematically in Figure 2. Since the transition from the state shown in Figure $2 \mathrm{a}$ to that in $2 \mathrm{~b}$ is gradual these two states may coexist for a relatively long time, the heavier current underneath having a counter clockwise circulation and the more diluted cloud above a clockwise circulation. This is a configuration called mixed avalanche. Note that the cloud above will begin to move faster than the heavier current below when it has attained sufficient mass.

\section{Experimental installation}

\section{I. The water tank}

The experimental set-up has already been briefly described in (I). It is a "Perspex" (polymethylmethacrylate) tank $50 \mathrm{~cm}$ high, $30 \mathrm{~cm}$ wide and $3 \mathrm{~m}$ long, which can be inclined up to $45^{\circ}$. A partition separates a length of $30 \mathrm{~cm}$ of this tank which is filled with brine (or 
in which the suspension is prepared); the rest of the tank is filled with fresh water. The two sections are connected with constant-head tanks, and a rotating vane, located in the partition near the floor, is used to control the injection of the brine.

\subsection{Measurement techniques}

A carriage is attached to the side of the channel which can be displaced with the front of the current or thermal. The position of this carriage as a function of time is recorded and this gives the front velocity. The growth rate of the gravity current is obtained from photographs taken with a camera mounted on this moving carriage. The current is made visible either by adding a small amount of Rhodamine B or adding tracer particles, and the whole flow or only a vertical section about $\mathrm{I} \mathrm{cm}$ wide can be illuminated. The velocity distribution is obtained by means of the hydrogen bubble technique.

The density distribution in the brine gravity current was measured by means of a oneelectrode conductivity probe. The sensitive element of this probe is $25 \mu \mathrm{m}$ in diameter and it measures the instantaneous resistance in a volume of about $250 \mu \mathrm{m}$ diameter.

\subsection{Operating procedure}

After the long and short sections of the tank have been filled respectively with fresh water and the brine, the channel is inclined and the static pressures are equilibrated. The rotating vane between the two sections is then opened and the pressure in the brine solution section is slightly increased so that the injection is critical to supercritical. The injection was either continuous or of short duration in which case only a head flow or thermal is established. In attempting to simulate the entrainment of snow, some experiments were run with a very thin layer of dense fluid (brine) distributed along the floor whose density was equal to or higher than the brine injected up-stream. In these experiments the tank was inclined after the opening of the vane. This distributed dense layer was only 2 to $3 \mathrm{~mm}$ thick and so its velocity remained small compared with the front velocity of the current.

\section{Experimental Results}

\section{I. Velocity and density profiles of a gravity current}

In (I) results showing the global development of inclined gravity currents and their relation to avalanche dynamics were given. Figures 3 and 4 show velocity and density profiles in such a current. It is seen that in the frontal zone of the head the velocity profile is similar to that of the steady layer and these profiles correspond to those proposed by de Quervain ( 1966$). *$ In the head, directly up-stream of the frontal zone and in the transition region the

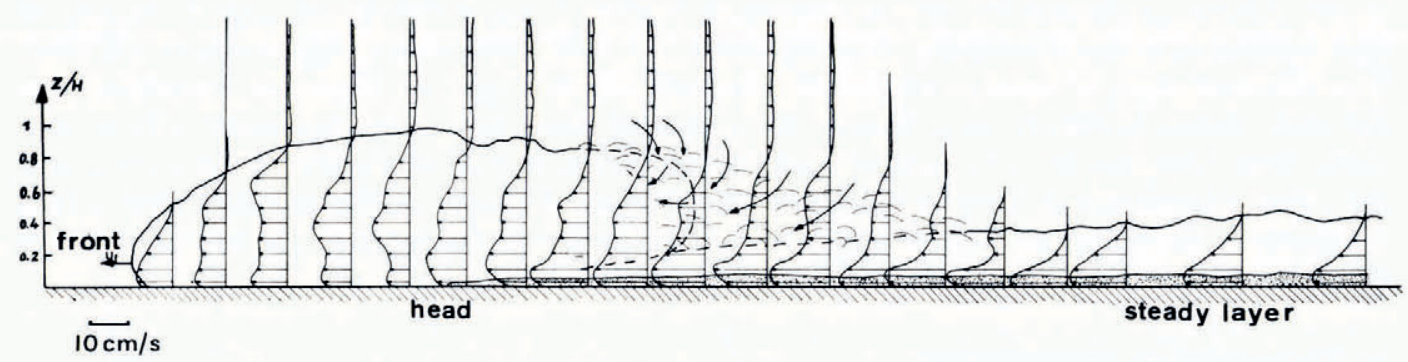

Fig. 3. Velocity profiles in a gravity current on a slope of $10^{\circ}$, with $\Delta \rho_{\mathrm{o}} / \rho_{0}=1 \%\left(U_{\mathrm{f}}=7 \mathrm{~cm} / \mathrm{s}\right)$. The shaded zone near the bottom indicates high density. Since the measurements have been made at a fixed position $x$ at constant time intervals, a distortion in height is introduced which for the current shown is $1.5 \mathrm{~cm}$ over the whole length, compared with $\mathrm{H}=10 \mathrm{~cm}$.

* Note that the velocity maximum just behind the nose is about $\mathrm{I} .5$ times the front velocity $U_{\mathrm{f}}$ and in the steady layer about $2.5 U_{\mathrm{f}}$. This relation may be somewhat slope dependent. 


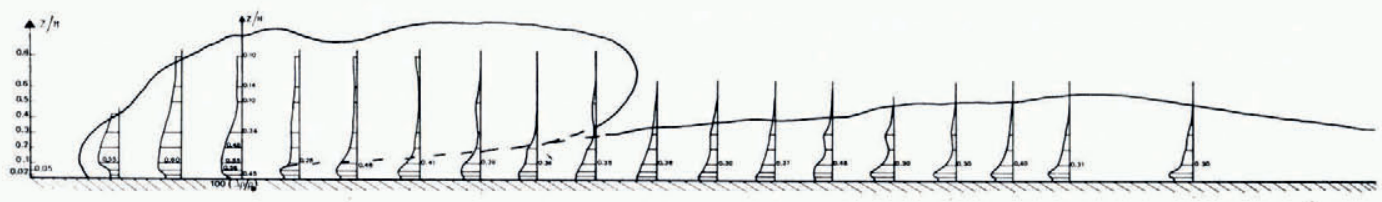

$$
\overline{-0.20} \cdots \cdot 1800=1
$$

Fig. 4. Density profiles in a gravity current on a slope of $10^{\circ}$ with $\Delta \rho_{0} / \rho_{0}=1 \%$. Note the penetration of the steady layer into the head and in particular the high density in the front, where the maximum is situated at about the nose height and its value is close to the density in the steady layer near the bottom. $H=10 \mathrm{~cm} . U_{\mathrm{f}} \simeq 7 \mathrm{~cm} / \mathrm{s}$.

profiles are, however, very irregular, indicating large-scale vortex-type motions. The profiles shown in Figure 3 have all been obtained from photographic records of the hydrogen bubble trains generated at one location $x$ at constant time intervals $\Delta \tau$. The interval $\Delta x$ shown in Figure 3 is given by $U_{\mathrm{f}} \Delta \tau$. The distortion in height from the front to the steady layer in Figure 2 is about $1.5 \mathrm{~cm}$ compared with $H=10 \mathrm{~cm}$.

The corresponding density profiles (Fig. 4) show that, except in the frontal zone of the head, the density is high close to the floor and falls off rapidly with height. In the front on the contrary the density maximum is situated approximately at the nose height and its value is close to the maximum density of the steady layer. These density distributions must be taken into consideration when calculating avalanche loads.

Records of the instantaneous density as a function of time at two heights are shown in Figure 5. From these records we can see that the density front is well defined but at $0 . \mathrm{I} H$ the density variations are very large in the frontal zone.

Figure 6(a) shows a vertical slice of the head of a gravity current under conditions practically identical to those of Figure 3. Aluminium particles have been added as markers and the photographs have been taken with a moving camera (moving with $U_{\mathrm{f}}$ ). The time of exposure was chosen such that each particle gives a trace which is tangent to the instantaneous streamlines; the characteristic streamline pattern of the flow structure is reproduced in Figure 6(b).

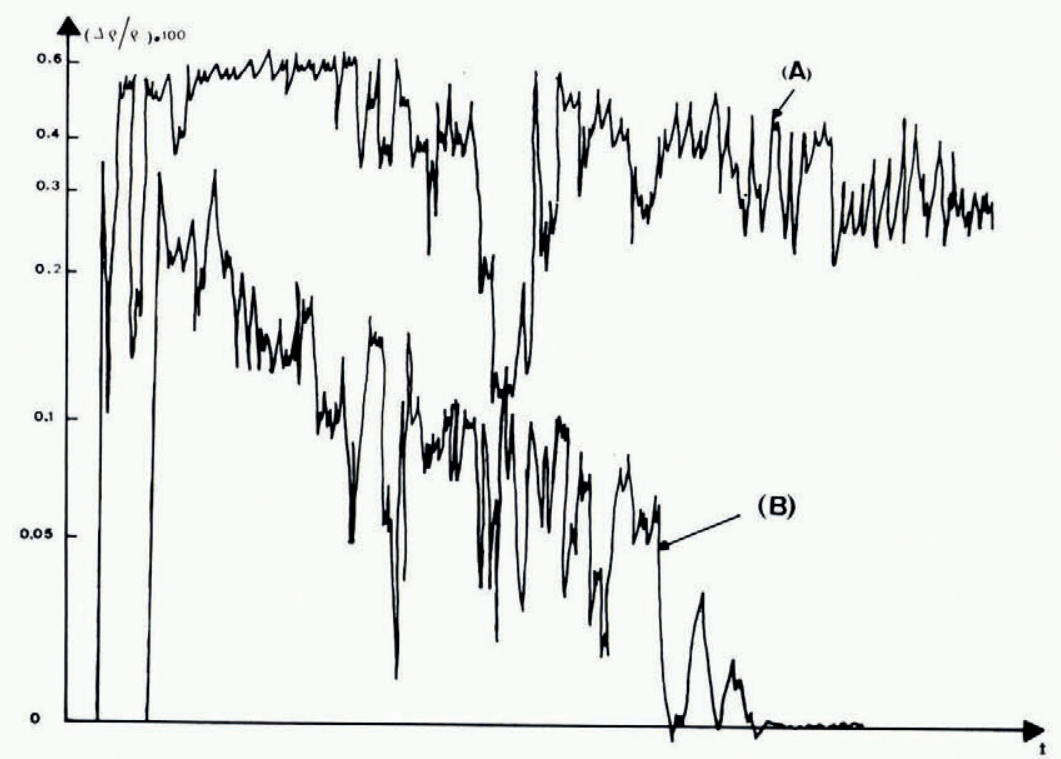

Fig. 5. Variation of the density with time at two heights in the current shown in Figure 3: $(A)$ at o.IH and $(B)$ at o.6H. These curves correspond to the signal of the conductivity probe located at a fixed position. 
In the frontal zone a big eddy of size $H$ is visible which moves the denser fluid from the wall outward and this explains the high density measured in the nose of a gravity current (Fig. 4). The eddy motion has some similarity with the flow field associated with a vortex pair, the difference being that here the vorticity is distributed over the whole region $H$ and the circulation is produced by buoyancy only. The lateral structure of an inclined gravity current shows lobes just as has been observed by Simpson (1972).

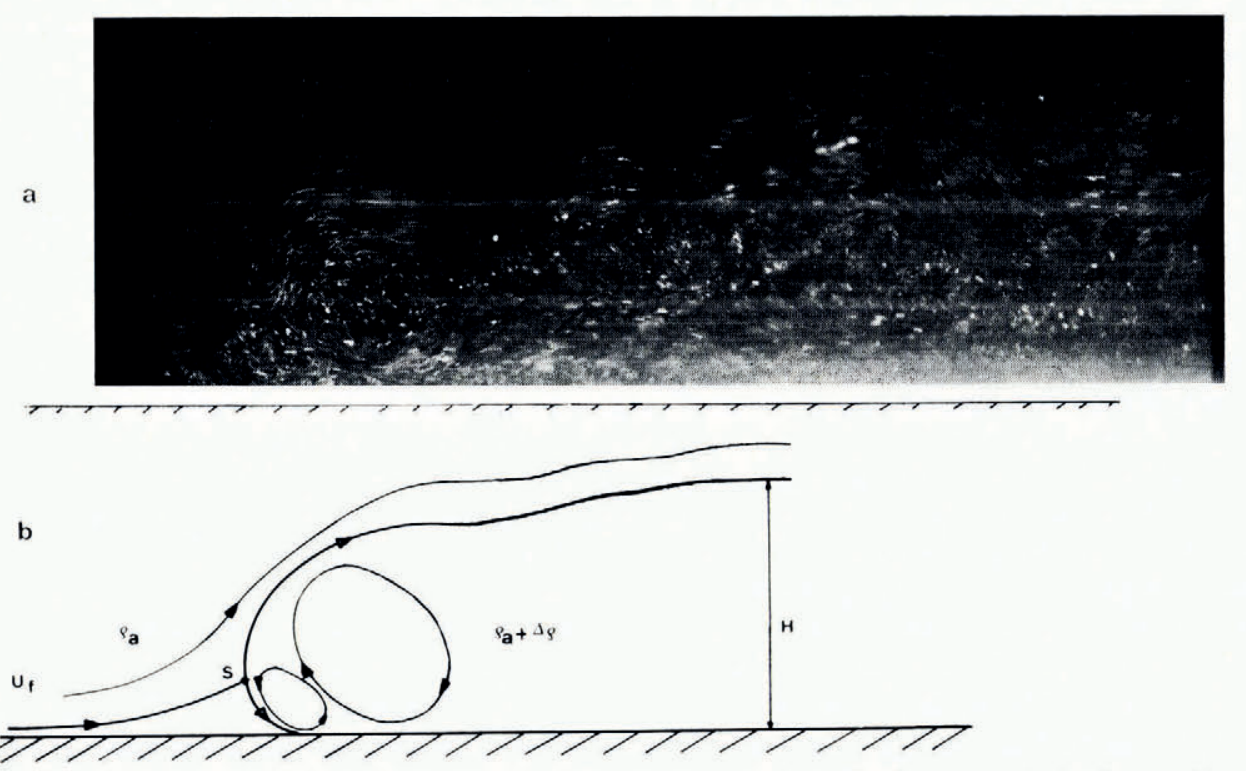

Fig. 6. View of a vertical slice of an inclined thermal containing tracer particles. The slope was $I 0^{\circ}, \Delta \rho_{0} / \rho_{0}=I \%$ and the observer is moving with the front. (a) photograph, $(b)$ instantaneous streamline pattern in which $S$ is the stagnation point. In all the photographs the flow is from right to left.

\subsection{Motion of an "inclined thermal"}

When only a finite volume of buoyant fluid is introduced, a thermal or a cloud (a head flow without a quasi-steady layer) develops whose velocity decreases with $x$ (see fig. 4 in (I)) and according to Equation (I0) it should vary as $U_{\mathrm{f}} \propto x^{-\frac{1}{2}}$. This predicted variation is in agreement with the experimental results (Fig. 7). These results yield $C \approx 2$ in Equation (I0) for the case of low density differences. The growth rate is roughly given by $\mathrm{d} H / \mathrm{d} x \approx$ $\approx 3 \times 10^{-3}(5+\alpha)$ ( $\alpha$ in degrees), and the front velocity is again practically independent of $\alpha$.

\subsection{Effect of a dense layer ahead of the front}

A very thin layer of density $\rho_{N}$ on the floor (simulating the powder-snow cover) ahead of the front may considerably modify the development of the flow. It is observed that this layer is to a large extent lifted up above the head and then is incorporated in the head by the turbulence resulting from a Kelvin-Helmholtz-type instability (Fig. 8). The nose of the current or thermal moves down into the thin layer. Experiments were run with a density $\Delta \rho_{\mathrm{N}}$ up to ${ }_{5} \Delta \rho_{0}$ and the same phenomenon was observed in all the experiments. According to Shoda (private communications) this seems to be indeed the way in which snow is entrained in a powder-snow avalanche. However, as pointed out in section 2.I, the structure of an avalanche at the start when $\bar{\rho} \approx \rho_{\mathrm{N}}$ may be quite different from this model and the snow is 


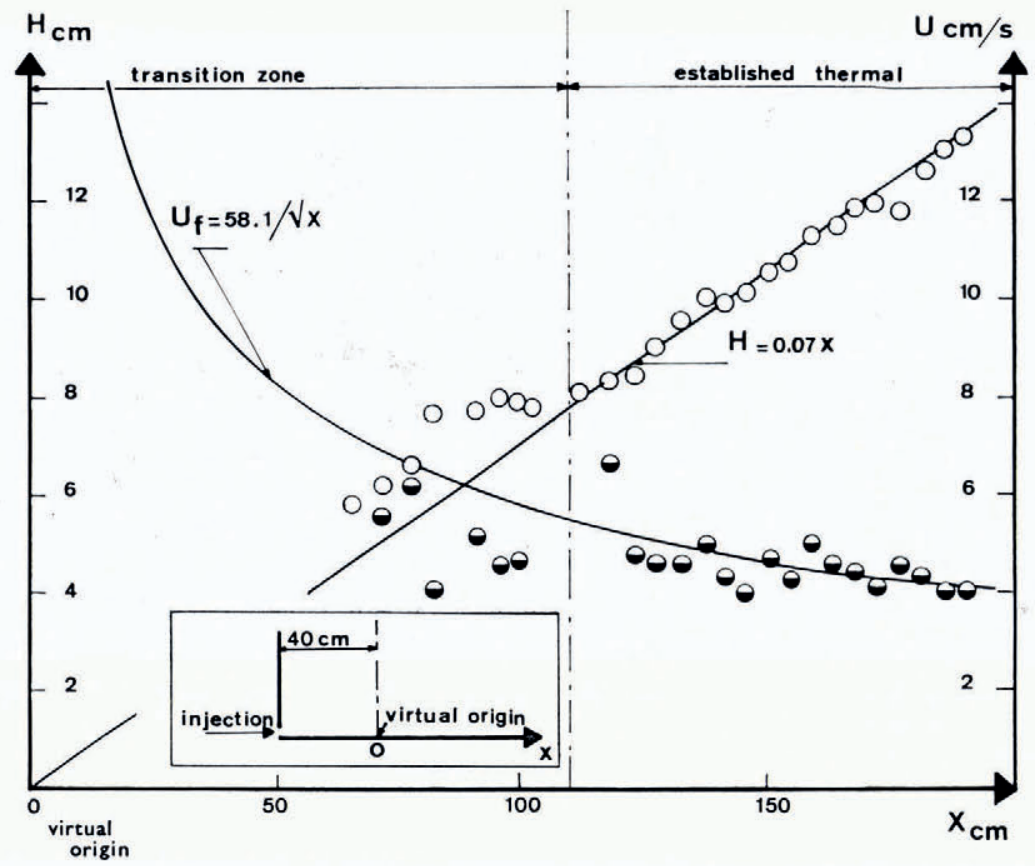

Fig. 7. Front velocity and growth rate of an inclined thermal on a slope of $20^{\circ}$ as a function of distance. $\Delta \rho_{0} / \rho_{0}=I \%$.

then likely to be entrained from underneath as the sense of the circulation of the frontal large eddy is probably counter-clockwise when the avalanche moves from right to left (the avalanche front erodes the snow cover (Fig. 2(a))).

The principal effect of the snow entrainment process as shown in Figure 8 is an increased front velocity and a decreased growth rate of the head; the distinction between the head aad the layer behind it seems to disappear. A thermal in the presence of such a dense layer of thickness $h_{\mathrm{N}}$ reaches a constant front velocity in agreement with Equation (9). This equation indicates that for large values of $x$ the velocity is $U_{\mathrm{f}} \propto\left(\Delta \rho_{\mathrm{N}} h_{\mathrm{N}}\right)^{\frac{1}{2}}$. When this entrainment mechanism is active a gravity current tends to become identical to an entraining thermal of $L \gg H$. Furthermore, when after a certain distance $x$ the dense layer suddenly stops, this structure changes into a gravity current.

\subsection{The effect of an obstacle}

Some experiments were run with an obstacle, representing a model of defence structures, mounted on the floor. This is a profiled obstacle with a wedge angle of $30^{\circ}$. Its height is about $\frac{1}{3}$ of the height of the head of the approaching current. Figure 9 shows that the obstacle causes an acceleration of the flow above it and then a vortex appears. This vortex acquires its circulation from the impulse due to the acceleration above the barrier.

Photographs of a vertical and horizontal slice of a gravity current containing tracer particles (represented respectively in Figure Io(a) and (b)) have permitted the determination of what could be called the "protected zone". This zone corresponds to the hatched region in Figure io. It is a relatively small region, and corresponds roughly to the obstacle dimensions, and it would not be free of snow deposit. 


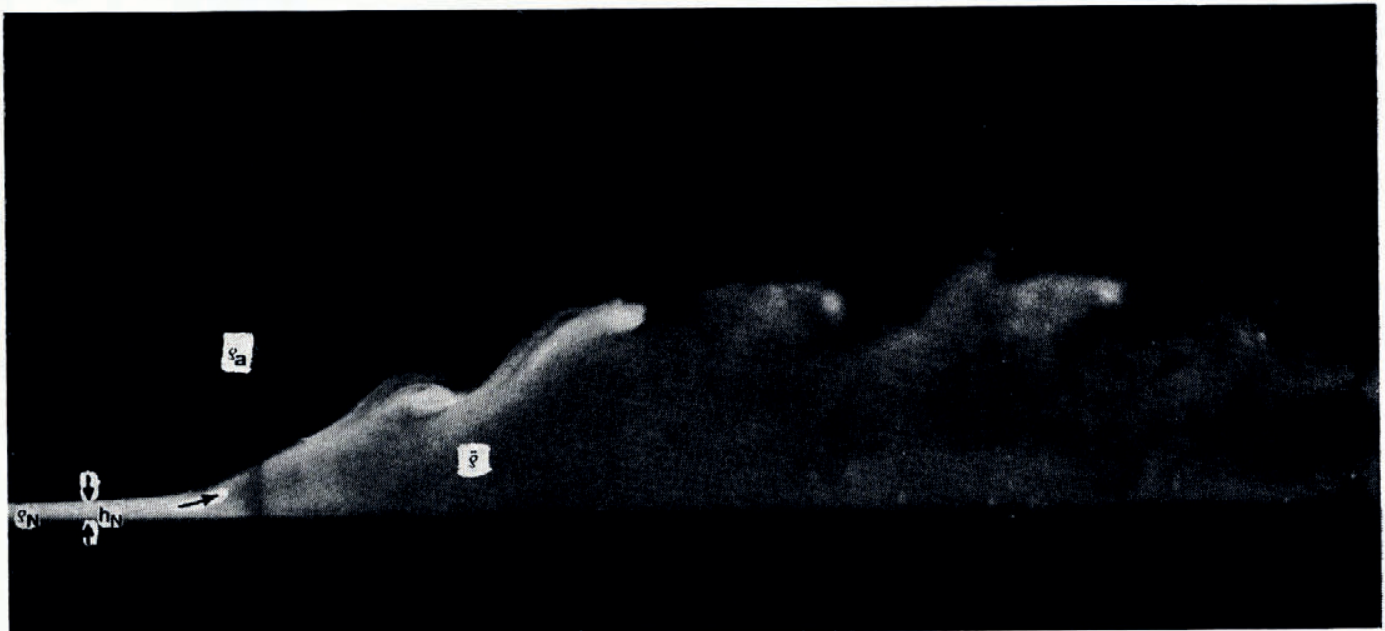

Fig. 8. View of a moving observer of a vertical slice of the head of a gravity current (or of a thermal) developing in the presence of a thin layer on the floor of density $\rho_{\mathrm{N}}=\rho_{0}\left(\Delta \rho_{0} / \rho_{0}=1 \%\right)$. It is seen that most of this thin dense layer is lifted up above the head. $\alpha=10^{\circ}$.

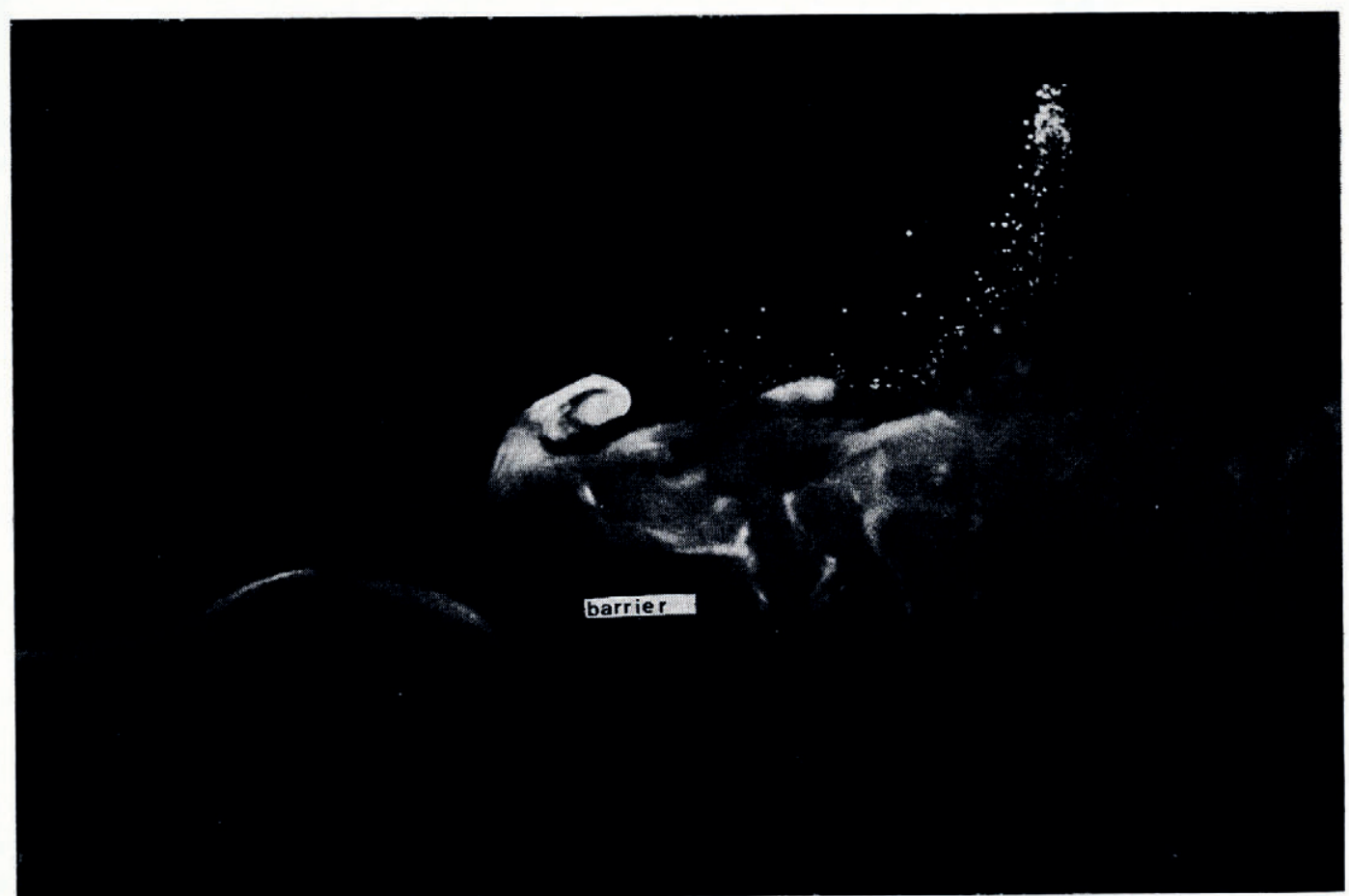

Fig. 9. View by a moving observer of a vertical slice of a gravity current when it traverses an obstacle of a wedge angle of $30^{\circ}$. The hydrogen bubble trains indicate an acceleration above the obstacle. Here, a dense layer was present on the floor, but the phenomena are the same without such a layer $\left(\Delta \rho_{0} / \rho_{0}=I \%, \alpha=I 0^{\circ}\right)$. 

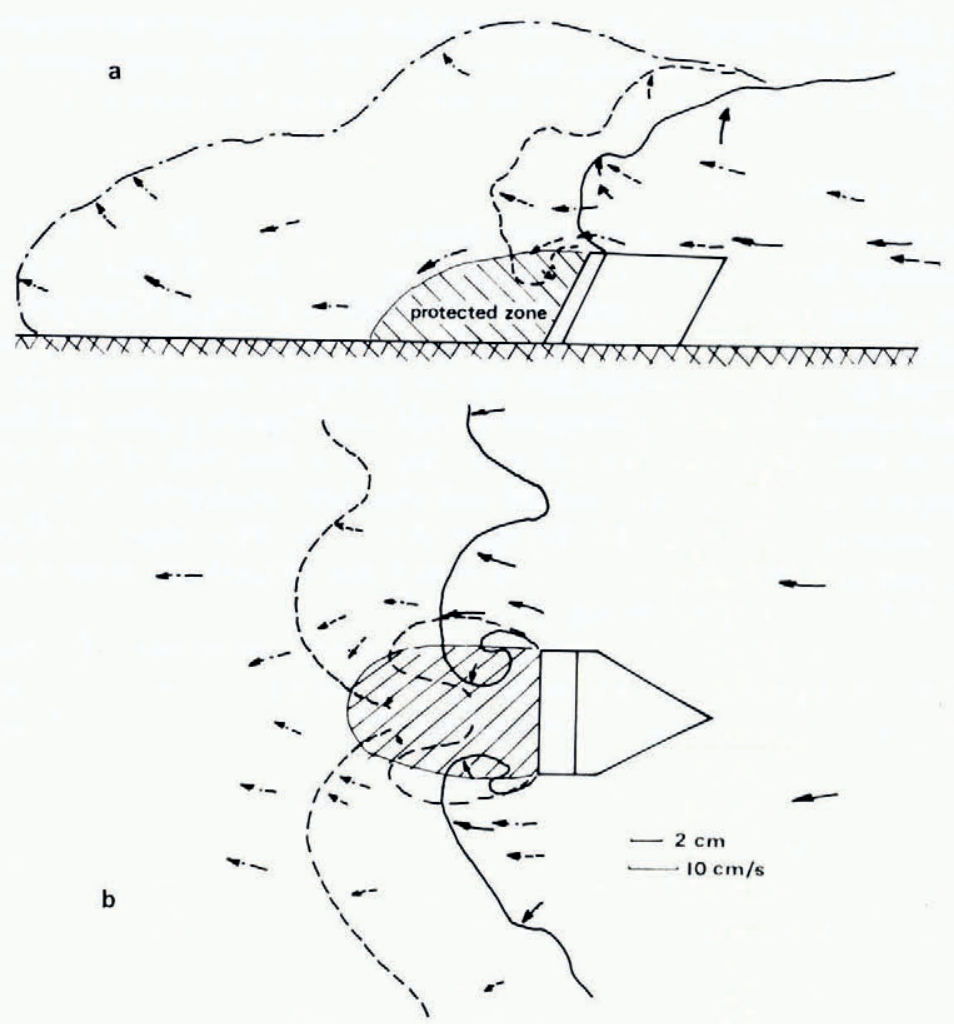

Fig. 1o. Approximate distribution of velocity when an avalanche front traverses an obstacle. (a) a side view, $(b)$ a top view. Note that $(a)$ and $(b)$ have been obtained from photographs of two different currents which explains the different positions of the front at time $T_{0}$. The hatched zone is the zone which can be considered to be protected but not free of snow deposit. $\alpha=10^{\circ}, U_{\mathrm{f}}=10 \mathrm{~cm} / \mathrm{s}, \longrightarrow$ at time $T_{0},---T_{0}+0.5 \mathrm{~s},-\cdots-\cdots T_{0}+2 \mathrm{~s}$.

\section{Practical applications}

As has been mentioned in the Introduction, the results obtained are strictly speaking valid only in situations where the Boussinesq approximation holds (sufficiently low density difference). Powder-snow avalanches rarely satisfy this approximation and it is, therefore, important to have a clear understanding of the inertial effects of large density variations. An attempt to express these effects quantitatively was made in (I), by assuming that the air entrainment rate is density independent. This assumption certainly needs to be verified but it is conjectured that any density effect on the entrainment rate will only result in a decreased growth rate of the avalanche and thus change the numerical value of the air drag. The internal structure, and hence the dynamics, will however remain similar to the low density situation.

The most reliable test of the laboratory simulation would be a comparison with field observation. From a qualitative viewpoint, we can say that photographs of powder-snow avalanches, like for instance figure I 9 shown by Voellmy (I955) indeed show a global structure identical to the laboratory current, that is to say the circulation of the frontal vortex is clockwise for the current moving from right to left. Furthermore, the front velocity of powder-snow avalanches is practically independent of the slope and seems to correlate well with $h_{\mathrm{N}}{ }^{\frac{1}{2}}$ (see figs. 24 and 25 in Voellmy, 1955). This behaviour is explained by the analysis and is in agreement with the experimental results presented in (I) and in this paper. Concerning the 
density distribution, Urumbayev (1974) observed that the density on trees struck by a so-called "air wave" is highest a certain distance above the ground. This distribution corresponds to the density profile in the front of a gravity current or a thermal (see Fig. 4).

\subsection{Evaluation of avalanche loads}

A knowledge of the avalanche load on structures is certainly of the greatest practical interest. We attempt here to give some guidelines on how the laboratory results can be used for this purpose. Most useful in this respect are the Figures 3 and 4 , which give us the velocity and density profiles from which we may deduce the dynamic pressure variations in an avalanche. From the velocity distribution shown in Figure 3 we can easily determine the non-dimensional velocity $U / U_{\mathrm{f}}$ as a function of $z / H$, and Figure 4 gives the density variation $\Delta \rho / \rho_{0}$ versus $z / H$ from which $\Delta \rho / \Delta \bar{\rho}\left(\Delta \bar{\rho} \equiv \bar{\rho}-\rho_{\mathrm{a}}\right)$, as a function of $z / H$ can be obtained. Because of dynamic similarity (internal Froude-number similarity) these reduced profiles correspond to the profiles in an avalanche when it is in the state shown in Figure 2(b). The scaling parameters are then $U_{\mathrm{f}}, \Delta_{\bar{\rho}}$ and $H$ which are themselves related by the internal Froude number $(F r)=U_{\mathrm{f}} /(g \Delta \bar{\rho} \cos \alpha H / \bar{\rho})^{\frac{1}{2}}$.

The problem then consists in determining these three parameters $\left(U_{\mathrm{f}}, \Delta \bar{\rho}\right.$ and $\left.H\right)$ for a given topography and given physical properties of the snow cover. The procedure is to calculate first $H$, which is practically independent of the density. Then $\Delta \bar{\rho}$ can be evaluated and finally $U_{\mathrm{f}}$.

\subsection{Height of an avalanche}

It was shown in (I) that the height of the quasi-steady layer depends primarily on the slope angle and is a linear function of $x$. It can be expressed in the form

$$
h=E x+h_{0},
$$

where $x$ is the distance travelled by the avalanche, $h_{0}$ is an initial height, and $E$ is approximately given by $E=10^{-3}(5+\alpha)$ ( $\alpha$ in degrees). A cloud of the form shown in Figure 2(d) also has a linear growth but the coefficient $E$ is closer to $3 \times 10^{-3}(5+\alpha)$. As has been shown in (I) the rate of growth of the head of a current of the type shown in Figure 3 depends somewhat on the conditions in the quasi-steady layer but in general $E \leqslant 3 \times 10^{-3}(5+\alpha)$. These are values obtained for low-density situations and it is likely that in a high-density flow the rate of growth is somewhat reduced (Townsend, r966).

\subsection{Average density}

In the evaluation of the average density of an avalanche, laboratory experiments are not of too much help; only the length and height of an avalanche cloud can be inferred from it. The average excess density is given by a relation of the form:

$$
\Delta \bar{\rho}=\Delta \rho_{\mathrm{N}} \frac{C_{2} h_{\mathrm{N}} L_{\mathrm{N}}}{S H L},
$$

where $C_{2}$ expresses the fractional height and $L_{\mathrm{N}}$ the length of the snow cover incorporated in the avalanche, $L$ is the avalanche length and $S$ a shape factor.

\subsection{Front velocity}

The front velocity can be calculated from Equation (10), generally assuming that snow is incorporated in the avalanche at a rate proportional to $U_{\mathrm{f}}$. Hence we use Equation (10) in the form:

$$
U_{\mathrm{f}}=C\left(g \frac{\sin \alpha}{E} C_{2} \frac{\Delta \rho_{\mathrm{N}}}{\bar{\rho}} h_{\mathrm{N}}\right)^{\frac{1}{2}} .
$$


For a low-density flow the constant $C$ has a value of about 2. Equation (12) in (I) indicates that for high-density flows $C$ will have a higher value or, which comes out to the same thing, the entrainment coefficient $E$ in Equation (13), must be weighted by $\rho_{\mathrm{a}} / \bar{\rho}$ because the momentum loss to the air entrained is weighted by the density ratio. Hence Equation (1 3 ) is used in the form:

$$
U_{\mathrm{f}}=C\left(g \frac{\sin \alpha}{E} C_{2} \frac{\Delta \rho_{\mathrm{N}}}{\rho_{\mathrm{a}}} h_{\mathrm{N}}\right)^{\frac{1}{2}} .
$$

\subsection{Dynamic pressure}

From Figures 3 and 4 it is possible to calculate the profiles of $\frac{1}{2} \rho U^{2}$. The corresponding stagnation pressure in an avalanche is then obtained by multiplying by the appropriate scales,

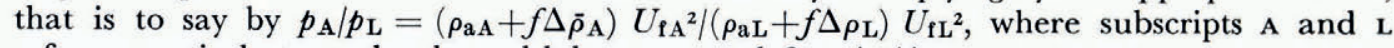
refer respectively to avalanche and laboratory and $f=\Delta \rho / \Delta \bar{\rho}$.

\subsection{Numerical example}

Assuming a mean slope angle $\alpha=30^{\circ}$ and a path length of $500 \mathrm{~m}$ we obtain:

$$
H \leqslant 50 \mathrm{~m} \quad \text { and } h=\mathrm{I} 8 \mathrm{~m} \text {. }
$$

Furthermore with $h_{\mathrm{N}}=\mathrm{I} \mathrm{m}$ and $\Delta \rho_{\mathrm{N}}=\rho_{\mathrm{N}}-\rho_{\mathrm{a}}=\mathrm{I}$ oo $\mathrm{kg} / \mathrm{m}^{3}$ the average density is: (with $C_{2}=\frac{1}{2}$ and $S=0.7$ )

$$
\Delta \rho \approx 0.035 \Delta \rho_{N} .
$$

Here we took $L={ }_{4} H$, which corresponds to laboratory observations, and the volume of any remaining steady layer was neglected. Note also that when the avalanche is in a state such as that shown in Figure $2 \mathrm{c}$ its height would correspond more to $h$ and hence $\Delta \bar{\rho} \lesssim$ o. I $\Delta \rho_{\mathrm{N}}$.

Using Equation (14) we obtain* as limits for the front velocity:

$$
80 \sqrt{ } h_{\mathrm{N}}<U_{\mathrm{f}}<150 \sqrt{ } h_{\mathrm{N}},
$$

depending on whether the avalanche is of height $H$ or $h$.

For the example considered, the maximum dynamic pressure to be expected is then $28 \mathrm{Mg} / \mathrm{m}^{2} \dagger$ when the avalanche is in the state shown in Figure $2(\mathrm{~b})$. Even when the avalanche is of the type shown in 2(c) the dynamic pressure is hardly higher, since in this case the front velocity is probably the maximum velocity of the avalanche.

\subsection{Non-stationary load}

There is another factor which may make an important contribution to avalanche loads on structures and which may explain loads of order $100 \mathrm{Mg} / \mathrm{m}^{2}$ which have sometimes been observed, namely the virtual-mass term. The load on a disc of diameter $a$ for example is composed of two terms:

$$
R(t)=\frac{\pi a^{2}}{4} \frac{\mathrm{I}}{2} \rho C_{\mathrm{D}} U^{2}+\frac{\pi a^{3}}{6} C_{\mathrm{m}} \rho \frac{\mathrm{D} U}{\mathrm{D} t},
$$

where $C_{\mathrm{D}}$ and $C_{\mathrm{m}}$ are the drag and inertia coefficients and have a value of order $\mathrm{I}$. The situation of an avalanche front hitting structures is somewhat similar to a wave front impinging on shore-line structures. The total time derivative $\mathrm{D} U / \mathrm{D} t$ may be taken $U_{\mathrm{f}} / a / U_{\mathrm{f}}$ and in this case the virtual mass term is of the same order as the drag term. Besides, when the density in the avalanche front is high compared with the air density a water-hammer phenomenon

\footnotetext{
* These values were obtained with $C=2$ in Equation (14). More extensive experiments have shown that $C$ is closer to $\mathrm{I}$ than 2.

$\dagger$ This is the value calculated with $U=2.5 U_{\mathrm{f}}$ and $\Delta \rho={ }_{4} \Delta \bar{\rho}$. In the frontal zone the pressure is closer to Io $\mathrm{Mg} / \mathrm{m}^{2}$ where $U \approx{ }_{1} .5 U_{\mathrm{f}}$.
} 
could occur in which case the non-stationary load would be proportional to $\rho_{\mathrm{f}} c U_{\mathrm{f}}$, where $c$ is the velocity of sound (in air most likely). This load, which can reach very high values (40 to $60 \mathrm{Mg} / \mathrm{m}^{2}$ ), would however be only of a duration $a / c$ which is very short, and concrete structures would probably resist such short-duration loads.

\section{Acknowledgement}

The authors gratefully acknowledge the financial support of this work by the Centre Technique du Génie Rural et des Eaux et Forêts, Grenoble.

\section{REFERENCES}

Ellison, T. H., and Turner, J. S. 1959. Turbulent entrainment in stratified flows. Fournal of Fluid Mechanics, Vol. 6, Pt. 3, p. $423-48$.

Longuet-Higgins, M. S., and Turner, J. S. 1974. An entraining plume model of a spilling breaker. Fournal of Fluid Mechanics, Vol. 63, Pt. 1, p. I-20.

Lovie, C., and others. 1974. Risques d'avalanche sur les gaves du glacier du Péclet Nord, Val Thorens, par C. Lovie, A. Col et J.-C. Lattes. C.E.R.N.A. [Centre d'Étude et Recherche sur la Neige et les Avalanches, Conflans, $73200-A l b e r t v i l l e]$. Rapport No. 5 .

Quervain, M. R. de. 1966. Problems of avalanche research. Union de Géodésie et Géophysique Internationale. Association Internationale d'Hydrologie Scientifique. Commission pour la Neige et la Glace. Division Neige Saissonière et Avalanches. Symposium international sur les aspects scientifiques des avalanches de neige, 5-10 avril 1965, Davos, Suisse, p. 15-22. (Publication No. 69 de l'Association Internationale d'Hydrologie Scientifique.)

Simpson, J. E. 1972. Effects of the lower boundary on the head of a gravity current. Fournal of Fluid Mechanics, Vol. 53 , Pt. 4, p. $759-68$.

Soo, S. L. 1967 . Fluid dynamics of multiphase systems. Waltham, Mass., Blaisdell Publishing Co.

Tochon-Danguy, J. C., and Hopfinger, E. J. [1975.] Simulation of the dynamics of powder avalanches. [Union Géodésique et Géophysique Internationale. Association Internationale des Sciences Hydrologiques. Commission des Neiges et Glaces.] Symposium. Mécanique de la neige. Actes du colloque de Grindelwald, avril 1974, p. 369-80. (IAHSAISH Publication No. 114.)

Townsend, A. A. 1966. The mechanism of entrainment in free turbulent flows. Fournal of Fluid Mechanics, Vol. 26, Pt. 4, p. 689-715.

Urumbayev, N. A. 1974. Rezul'taty issledovaniy prirody i deyatel'nosti lavinnykh vozdushnykh voln. (In Moskalev, Yu. D., ed. Glyatsiologiya Sredney Azii. Laviny [Glaciology of Central Asia. Avalanches]. Sredneaziatskiy Regional'nyy Nauchno-Issledovatel'skiy Gidrometeorologicheskiy Institut. Trudy, Vyp. ${ }^{15}(96)$, p. $\left.31-38.\right)$

Voellmy, A. 1955. Über die Zerstörungskraft von Lawinen. Schweizerische Bauzeitung, Jahrg. 73, Ht. 12, p. $159-62$; Ht. 15, p. 212-17; Ht. 17, p. 246-49; Ht. 19, p. 28o-85. 